\title{
Estudio caso-control de sangrados mayores cerrados en pacientes descoagulados con acenocumarol y heparina-no fraccionada
}

\author{
J. MARTÍNEZ LACASA, N. JUAN, J. JULIÁ, M. RODRÍGUEZ-CARBALLEIRA, \\ I. DE DIEGO ${ }^{1}$, R. SOTO ${ }^{1}$, J. GARAU
}

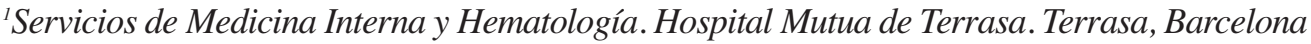

\section{RESUMEN}

Objetivos: Determinar las características de los episodios de sangrado mayor cerrado (SM). Valorar los controles de la anticoagulación mediante el INR del tiempo de protrombina (TP) y el tiempo de tromboplastina parcial (TTPA) durante los episodios. Analizar la mortalidad directamente relacionada con los episodios de SM. Comparar estos factores con un grupo control.

Métodos: Estudio caso-control de los SM registrados en la base de datos de monitorización de los tratamientos anticoagulantes de nuestro centro desde enero de 1995 hasta diciembrede 2002. Se define caso como cualquier episodio de SM en paciente que recibiese tratamiento anticoagulante y control como cualquier paciente sin episodio previo o actual de SM al que se realizó una determinación de INRo TTPA el mismo día que el caso de SM, siempre que estuvieran emparejados por edad, sexo, tipo e indicación de tratamiento anticoagulante.

Resultados: Análisis descriptivo: Desde enero 1995 a diciembre de 2002 se estudiaron de forma prospectiva 225 pacientes en tratamiento anticoagulante, de los cuales 75 fueron casos de SM y 155 controles. Durante este periodo se controlaron un total de 1650 pacientes por lo que la prevalencia de SM fue del 4,5\%. Los motivos de descoagulación del total de 225 pacientes fueron: fibrilación auricular en 79 pacientes $(35,3 \%)$, valvulopatías en $59(25,9 \%)$, TVP-TEP en $48(21,4 \%)$, miocardiopatía dilatada o isquémica en $26(11,6 \%)$ y AVC en $13(5,8 \%)$. La edad media de los casos fue de 70,5 años (DE 9,5) siendo mujeres 41 (55\%). En el momento del sangrado 39 casos (52\%) seguían tratamiento con TAO y 36 casos (48\%) estaban tratados con HS. El INR medio en el momento del sangrado fue de 5,3 (DE 7,5) y el TTPA de 2,25 (DE 0,95). Tenían antecedentes de sangrado previo $24(32 \%)$ de los casos. La localización del SM fue por orden de frecuencia: muscular (40\%), sistema nervioso central $(30,6 \%)$, retroperitoneal $(18,6 \%)$ y articular $(10,6 \%)$. Los pacientes tratados con TAO sangraron significativamente con más frecuencia en SNC mientras que los pacientes en tratamiento con HS lo hicieron en músculo o retroperitoneal ( $\mathrm{p}<0,0001)$. Murieron a causa del SM 11/75 casos $(14,6 \%)$ y por otras razones 3 casos (4\%). Análisis comparativo: El INR de los casos estaba dentro del rango terapéutico en el momento del sangrado en el $38.5 \%$ respecto del 75,3\% de los controles $(\mathrm{p}<0,0001)$. Se observaron diferencias significativas entre el valor de INR medio de casos $(5,3 \pm 7,5)$ y de controles $(2,5 \pm 0,95)$ con una $\mathrm{p}<0,001 \mathrm{y}$ no las hubo entre el TTPA de casos y controles. En el 32\% de los casos existía un episodio de sangrado previo, antecedente que sólo tenían el $1,3 \%$ de los controles ( $p<0,001)$ siendo éste un factor de riesgo independiente para un nuevo sangrado $(\mathrm{RR}=34,5)$. La mortalidad global de los casos fue del 18,6\% (14/75) mientras que en los controles fue del 11,4\% $(18 / 155) ; \mathrm{p}=0,01$.

Conclusiones: En nuestro estudio los casos de SM tratados con TAO tiene controles de INR(TP) fuera de su rango terapéutico en una proporción mayor que los casos tratados con HS. El sangrado previo es un im-
MAJOR CLOSE SPACE BLEEDING IN PATIENTS ON ANTICOAGULATION WITH ACENOCUMAROL (TAO) OR NON FRACTIONNED HEPARIN (HS): A CASE-CONTROL STUDY

\section{ABSTRACT}

Objetive: To study the characteristics of major bleeding episodes into a closed space (BCS) of patients under chronic anticoagulation with either unfractionated heparin $(H S)$ or coumadin $(C M)$, and to determine the relationship, if any, of anticoagulation parameters (INR, PT and PTT) values at the time of bleeding with the episode. Finally, to determine risk factors for BCS and mortality in this population.

Methods: Descriptive epidemiology of all cases of BCS seen in our hospital from 1995 to 2000 was obtained through the records and follow up visits of all patients under anticoagulation (HS or CM) during this period. A matched case-control study to determine risk factors for BCS was carried out. Cases and controls (1:2) were matched for age, gender, anticoagulant treatment and indication for anticoagulation. Cases were patients with a BCS while on anticoagulation (HS OR CM). Controls were patients under anticoagulation (HS or CM) without any bleeding episode during the study period that had anticoagulation parameter values (INR, PT or PTT) determined the very same day than the cases.

Results: During the study period, 225 patients under anticoagulation were prospectively followed ( 75 cases and 150 controls) amid a total of 1650 patients under anticoagulation, for a $4.5 \%$ prevalence of BCS. Reasons for anticoagulation were: atrial fibrillation in 79 (35.3\%), valvular heart disease in $59(25.9 \%)$, pulmonary embolism or deep venous thrombosis in 48 (21.4\%), dilated cardiomyopathy in 26 (11.6\%) and vascular cerebral stroke in $13(5.8 \%)$. Mean age of cases was 70.5 (SD 9.5) years and $41(55 \%)$ were women, values similar to the controls. At the time of BCS 39 patients were on CM and 36 on HS. The mean INR value in the $C M$ group at the time of the episode of BCS was $5.3(S D+7.5)$ while the $P T T$ value was 2,25 (SD 0.95) in the HS group. There was previous antecedent bleeding in $24(32 \%)$ cases. The most common sites of BCS were: muscular (40\%), CNS (30.6\%), retroperitoneal (18,6\%) and articular (10.6\%). Muscular (abdominal or thoracic wall) and retroperitoneal $B C S$ were higher in the HS group (10 and 12 in the HS group versus 5 and 2 in the CM group, respectively; $p<0.0001)$. In contrast, CNS bleeding was commoner in the CM group (20 in CM versus 3 in $H S ; p<$ $0.001)$. BCS related mortality rate was $14.6 \%$ (11/75) and higher in the CM group $(p=0.04)$. Comparative analysis of the case-control study revealed that anticoagulation values in the CM group at the time of bleeding were within the recommended range in $38.5 \%$ of cases vs. $75 \%$ of the controls $(p<0.001)$. Also, there were significant differences in mean INR values between cases and controls $(5.3+7.5$ vs. $2.6+0.9, p<$ $0.029)$ In the HS group no differences were present in PTT values at the time of bleeding between cases and controls. In BCS cases, a previous bleeding episode was more frequent than in the control group (32\% versus $1.3 \%, p<0.001)$. Likewise, mortality was higher in cases $(18,6 \%)$ than in controls (11.4\%), $p=0.01$. 
portante factor de riesgo para SM. El SM por TAO es tardío en el curso del tratamiento y predomina los episodios de sangrado a nivel del SNC mientras que en los pacientes tratados con HS el sangrado es a nivel muscular o retroperitoneal. La mortalidad directamente relacionada con el SM es del 14,6\%. Una cuidadosa monitorización del INR del tiempo de protrombina se revela como única estrategia para disminuir el riesgo de sangrado.

PALABRAS CLAVE: Episodios de sangrados cerrados. Pacientes descoagulados. Acenocumarol. Heparina no fraccionada.
Conclusions: In our study, the majority of patients under anticoagulation with CM had INR values above the recommended range at the time of BCS, in contrast with those on HS that had a PTT within the therapeutic range at the time of the BCS. A previous bleeding episode was an independent risk factor for a BCS episode. Bleeding was a late complication in the CM group and frequently in the CNS, while BCS was more frequently associated with muscular or retroperitoneal sites in the $H S$ treated group. BCS related mortality was $15 \%$. Close monitoring of INR is crucial to minimize bleeding complications.

KEY WORDS: Major bleeding episodes. Patients anticoagulation. Acenocumarol. Unfractionated heparin.

Martínez Lacasa J, Juan N, Julià J, Rodríguez-Carballeira M, de Diego I, Soto R, Garau J. Estudio caso-control de sangrados mayores cerrados en pacientes descoagulados con acenocumarol y heparina-no fraccionada. An Med Interna (Madrid) 2008; 25: 9-14.

\section{INTRODUCCIÓN}

En las últimas décadas el tratamiento anticoagulante se ha generalizado en diversos grupos de pacientes tanto para la prevención primaria o secundaria de complicaciones tromboembólicas en pacientes con alteraciones de ritmo cardiaco y/o valvulopatías, como en el tratamiento de la trombosis venosa profunda o la embolia pulmonar. Con frecuencia ambos procesos suponen una terapia anticoagulante a largo plazo.

El riesgo de sangrado es la principal complicación del tratamiento anticoagulante en esta población (1-3). Los sangrados pueden ser cerrados o abiertos; estos últimos tienen una expresión clínica mucho más evidente. Su diferenciación és útil tanto por la presentación clínica como por la morbimortalidad que conlleva uno y otro tipo de sangrado.

Los diversos estudios realizados para identificar los factores de riesgo de sangrado no han permitido aclarar la polémica sobre este tema (4-7). Así, algunos han encontrado una clara tendencia a las complicaciones hermorrágicas en los pacientes ancianos mientras que otros descartan la edad como factor de riesgo independiente $(4,5)$. La existencia de comorbilidad incluyendo a la fibrilación auricular, el accidente vascular cerebral, la diabetes mellitus, el fallo cardiaco crónico, el cancer o la úlcera péptica son factores de riesgo que, asociados a descoagulación, aumentan el riesgo de sangrado, según otros6. Esta afirmación llama la atención ya que algunas de estas enfermedades son precisamente las causas más frecuentes de indicación de la anticoagulación.

A partir del trabajo de Van der Meer8 que diseñó el denominado índice de riesgo de sangrado se pudo observar que en los pacientes que recibían tratamiento anticoagulante crónico con TAO la variabilidad de los controles del tiempo de protrombina tenía influencia en el riesgo de sangrado, de forma que variaciones elevadas por encima de las ratios recomendadas predecían el riesgo de sangrado. La incidencia de complicaciones hemorrágicas fue inversamente proporcional a la duración de la anticoagulación. Si bien otros estudios no han confirmado estos datos, Casais $\mathrm{P}$ y cols. $(9,10)$ concluyeron en su estudio que la intensidad de la anticoagulación en referencia a su duración y la desviación de los controles de INR respecto de las ratios recomendadas son los factores de riesgo asociados de forma más evidente al sangrado.
Con la pretensión de profundizar en el conocimiento de los factores de riesgo de sangrado cerrado mayor (SM) en los pacientes sometidos a anticoagulación bien con heparina sódica (HS) o bien con acenocumarol oral (TAO), hemos realizado un estudio prospectivo y caso-control, incluyendo todos los casos de SM observados en nuestro hospital durante un periodo de 7 años.

\section{MATERIAL Y MÉTODOS}

Analisis prospectivo, comparativo y caso-control de todos los episodios de sangrado mayor cerrado observados en nuestro hospital en pacientes en tratamiento anticoagulante con TAO o bien con HS.

\section{DEFINICIONES}

Se consideró sangrado mayor cerrado (SM) cuando se constataba un sangrado no abierto hacia el exterior con repercusión clínica o analítica en cualquier paciente en tratamiento anticoagulante con TAO o HS. El diagnóstico de sangrado se realizó por criterios clínicos, analíticos y mediante técnicas de imagen.

Se definió sangrado previo como el antecedente de un sangrado (abierto o cerrado) con repercusión clínica en pacientes en tratamiento anticoagulante antes de la inclusión de su en el estudio.

Casos fueron definidos como cualquier paciente en anticoagulación crónica que presentó un SM durante el periodo de anticoagulación.

Controles fueron aquellos pacientes en anticoagulación crónica que tuvieron un control de anticoagulación el mismo día que un caso, ajustados por edad, sexo, tipo de anticoagulación y motivo de la misma.

Los controles de la ratio de anticoagulación se realizaron siempre con el mismo criterio y con la misma periodicidad en todos los casos. El control de anticoagulación de los pacientes tratados con TAO se realizó mediante el INR del tiempo de protrombina (INR), mientras que los pacientes tratados con HS se controlaron mediante el tiempo de tromboplastina parcial activado (TTPA). 


\section{ESTUDIO DESCRIPTIVO}

Se incluyeron todos los pacientes controlados en nuestro hospital durante los años 1995-2002 en tratamiento anticoagulante por cualquier causa y que tuvieran un seguimiento mínimo de una semana. Se analizaron todos los pacientes que presentaron un SM durante el periodo del estudio y en tratamiento anticoagulante con TAO o con HS.

Se estudiaron diversas variables relacionadas con el episodio de sangrado y la evolución de los casos y que incluían junto a los datos demográficos de edad, sexo y comorbilitad, el motivo de la anticoagulación, el tipo de la misma, los controles de INR y TTPA en el momento del episodio, la localización y la evolución de los SM (Tabla I). Se prestó especial interés a aquellos parámetros que podrían constituir factores de riesgo de sangrado como los tratamientos concomitantes, la ratio del INR o el TTPA en el momento del episodio y la historia de sangrado previa. Respecto a dichas variables y especialmente la localización del sangrado y su evolución, se realizó un estudio univariante entre los pacientes anticoagulados con TAO y HS (Tablas I y II).

\section{ESTUDIO COMPARATIVO}

El estudio caso-control se realizó asignando 2 controles por caso entre aquellos pacientes a los que se realizó un control de coagulación en el mismo día que se registró el episodio de SM. Se ajustaron los controles por edad, sexo, indicación de descoagulación y tratamiento con TAO o con HS.

Se siguieron de forma prospectiva los casos y los controles hasta el final del tratamiento anticoagulante por cualquier motivo (aparición de un SM, fin del estudio, finalización de anticoagulación o bien la muerte del paciente). Los pacientes fueron analizados y seguidos de manera prospectiva en una base de datos de la sección de hemostasia del servicio de Hematología que contiene los datos analíticos de las ratios de control tanto de INR como TTPA en cada control periódico, así como los diferentes eventos clínicos.

En el estudio comparativo las variables cuantitativas fueron estudiadas mediante el test de la t de Student mientras que las variables cualitativas se analizaron mediante el test del Jicuadrado.

El tiempo hasta el sangrado en el grupo de los casos y la mortalidad se analizaron mediante una curva de Kaplan-Meier, (Figs. 1 y 2).

\section{RESULTADOS}

\section{ESTUDIO DESCRIPTIVO GLOBAL}

Entre enero de 1995 y diciembre de 2002 se contabilizaron en la base de datos de hemostasia 1650 pacientes en tratamiento anticoagulante. Durante este periodo se registraron 75 episodios de SM, lo que supone una prevalencia del 4,5\%. Estos episodios mas sus respectivos controles (150 pacientes), fueron objeto del presente análisis.

De los 225 casos y controles la edad media fue de 68 años $(\mathrm{DE} \pm 10,1)$. Las indicaciones del tratamiento anticoagulante fueron: prevención de las complicaciones embólicas en la arritmia completa por fibrilación auricular (ACxFA) en 79 casos (35\%) valvulopatía en 59 (26\%), trombosis venosa profunda y/o embolia pulmonar en 48 (21\%), miocardiopatía en $26(12 \%)$ y finalmente accidente vascular cerebral (AVC) con criterio de descoagulación en $13(6 \%)$.

TABLA I

CARACTERÍSTICAS GENERALES Y VARIABLES RELACIONADAS CON EL SM DE PACIENTES. ANÁLISIS UNIVARIANTE DE CASOS Y CONTROLES

\begin{tabular}{|c|c|c|c|c|c|}
\hline Variables & $\begin{array}{l}\text { Total } \\
(n=225)\end{array}$ & $\begin{array}{l}\text { Casos } \\
(n=75)\end{array}$ & $\begin{array}{l}\text { Controles } \\
(n=150)\end{array}$ & p & OR-IC95\% \\
\hline $\begin{array}{l}\text { Edad } \\
\text { Sexo hombres/mujeres } \\
\text { Motivo de anticoagulación }\end{array}$ & $\begin{array}{c}70 \pm 10 \\
98(44 \%) / 126(56 \%)\end{array}$ & $\begin{array}{c}71 \pm 10 \\
34(45 \%) / 41(55 \%)\end{array}$ & $\begin{array}{c}70 \pm 10 \\
65(43 \%) / 85(57 \%)\end{array}$ & $\begin{array}{l}0,849 \\
0,73\end{array}$ & $1,1(0,6-1,9)$ \\
\hline $\begin{array}{l}\text { Valvulopatía } \\
\text { FA } \\
\text { AVC } \\
\text { TVP-TEP } \\
\text { Miocardiopatía dilatada }\end{array}$ & $\begin{array}{l}59(25 \%) \\
79(35 \%) \\
13(6 \%) \\
48(21 \%) \\
26(12 \%)\end{array}$ & $\begin{array}{l}14(19 \%) \\
25(33 \%) \\
5(7 \%) \\
21(28 \%) \\
10(13 \%)\end{array}$ & $\begin{array}{l}44(30 \%) \\
54(36 \%) \\
8(5 \%) \\
27(18 \%) \\
17(11 \%)\end{array}$ & 0,27 & \\
\hline $\begin{array}{l}\text { Anticoagulante } \\
\text { Heparina }\end{array}$ & $104(46 \%)$ & $36(48 \%)$ & $69(46 \%)$ & 0,73 & $0,9(0,5-1,5)$ \\
\hline $\begin{array}{l}\text { Dicumarínicos } \\
\text { Tiempo hasta el sangrado (días) }\end{array}$ & $120(54 \%)$ & $\begin{array}{c}39(52 \%) \\
\text { Media: } 768 \pm 134 \\
\text { Mediana: } 570\end{array}$ & $\begin{array}{c}81(54 \%) \\
\text { Media: } 358 \pm 122 \\
\text { Mediana :7 }\end{array}$ & & \\
\hline Tiempo seguimiento (días) & $\begin{array}{l}\text { Media: } 1252 \text { (1167) } \\
\quad \text { Mediana: } 961\end{array}$ & $\begin{array}{l}\text { Media:961 (1105) } \\
\text { Mediana: } 364\end{array}$ & $\begin{array}{l}\text { Media: } 1400(1173) \\
\text { Mediana: } 1173\end{array}$ & 0,006 & \\
\hline $\begin{array}{l}\text { Sangrado previo } \\
\text { TTPA } \\
\text { TTPA anormal } \\
\text { INR } \\
\text { INR anormal }\end{array}$ & $\begin{array}{c}26(12 \%) \\
2,13 \pm 0,92 \\
27(12 \%) \\
3,48 \pm 4,51 \\
44(20 \%)\end{array}$ & $\begin{array}{l}24(32 \%) \\
2,5 \pm 0,9 \\
12(32 \%) \\
5,3 \pm 7,5 \\
24(62 \%)\end{array}$ & $\begin{array}{c}2(1,3 \%) \\
2 \pm 0,9 \\
15(22 \%) \\
2,6 \pm 0,9 \\
20(25 \%)\end{array}$ & $\begin{array}{c}<0,001 \\
0,338 \\
0,25 \\
0,029 \\
<0,001\end{array}$ & $34,5(8-151)$ \\
\hline
\end{tabular}

FA: fibrilación auricular; AVC: accidente vascular cerebral; TVP-TEP: trombosis venosa profunda-tromboembolismo pulmonar. 
TABLA ॥

ANÁLISIS DE LOS EPISODIOS DE SANGRADO. LOCALIZACIÓN Y EVOLUCIÓN* CLÍNICA. ESTUDIO UNIVARIANTE

\begin{tabular}{|c|c|c|c|c|c|}
\hline & $\begin{array}{l}\text { Totales } \\
(n=75)\end{array}$ & $\begin{array}{c}\text { Dicumarínicos } \\
\quad(n=39)\end{array}$ & $\begin{array}{l}\text { Heparina } \\
(n=36)\end{array}$ & $p$ & OR-IC95\% \\
\hline \multicolumn{6}{|l|}{ ELocalización } \\
\hline Pared tórax & $3(4 \%)$ & $2(5 \%)$ & $1(2,7 \%)$ & 1 & 1,$8 ; 0,16-21$ \\
\hline Retroperitoneal & $14(18,6 \%)$ & $2(5 \%)$ & $12(31 \%)$ & 0,009 & 0,$12 ; 0,02-0,67$ \\
\hline Pared abdominal & $12(16 \%)$ & $3(7,6 \%)$ & $9(33,3 \%)$ & 0,002 & 0,$12 ; 0,03-0,9$ \\
\hline Fascias EEII & $5(6,6 \%)$ & $3(7,6 \%)$ & $2(5,6 \%)$ & 0,4 & 2,$5 ; 0,38-13$ \\
\hline \multicolumn{6}{|c|}{ Evolución del sangrado } \\
\hline Reabsorción & $55(73,3 \%)$ & $28(71,7 \%)$ & $27(75 \%)$ & 0,79 & $0.8 ; 0,3-3$ \\
\hline Cirugía & $9(12 \%)$ & $3(7,6 \%)$ & $6(16,6 \%)$ & 0,29 & 0,$4 ; 0,07-2,11$ \\
\hline Exitus & $11(14,6 \%)$ & $8(20,5 \%)$ & $3(8,3 \%)$ & 0,19 & 2,$8 ; 0,6-15$ \\
\hline
\end{tabular}

${ }^{*}$ Muscular: fascias + pared abdominal + pared torácica.

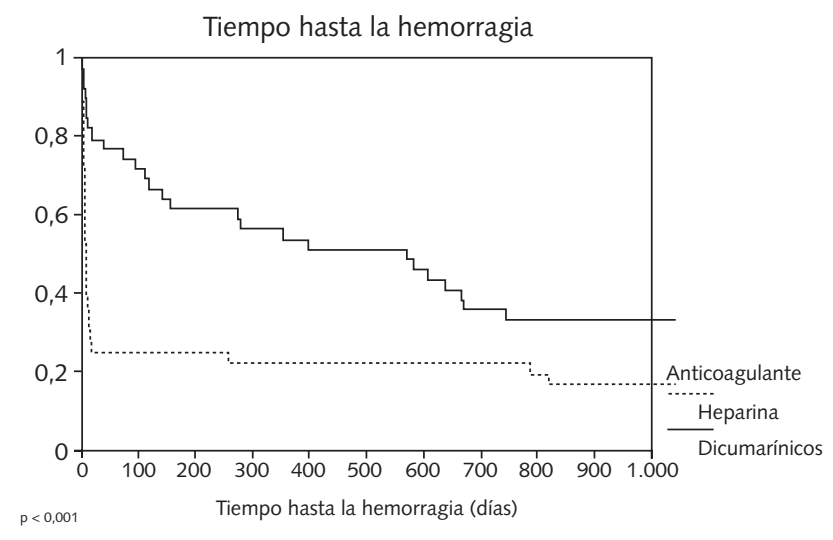

Fig. 1. Curva de Kaplan-Meier: tiempo hasta sangrado.

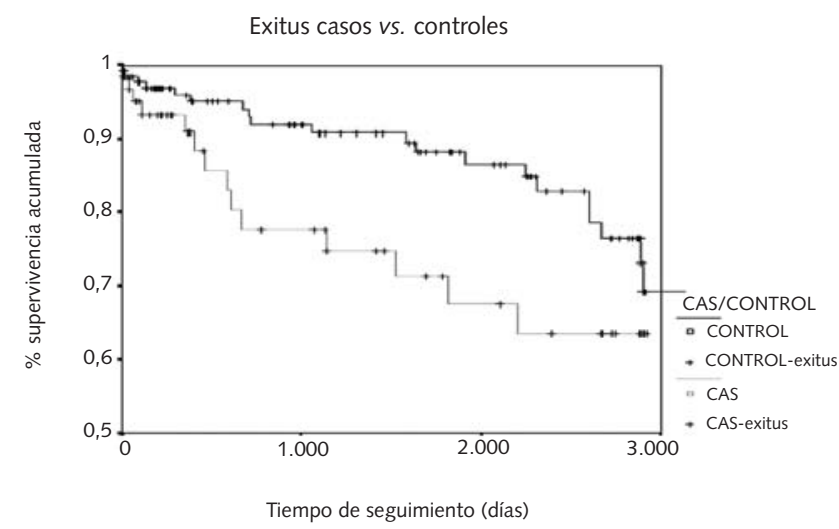

Fig. 2. Curva de Kaplan-Meier: mortalidad entre casos y controles.
En la tabla I se especifican las causas de anticoagulación de los casos y los controles...

\section{Análisis de los casos: SM, 75 pacientes}

La edad media de los pacientes que presentaron un SM fue de 70,5 años (DE \pm 10$)$ siendo un $55 \%$ mujeres. $39(52 \%)$ pacientes estaban anticoagulados con TAO mientras que los 36 casos restantes estaban con HS. Un $32 \%$ de los casos de SM tenían un antecedente previo de sangrado con una media de 16 meses (DE $\pm 3,2) .14$ pacientes murieron, siendo la mortalidad directamente atribuible al SM en 11 casos (14,6\%).

La localización del SM fue por orden de frecuencia; muscular $(40 \%)$, sistema nervioso central $(30,6 \%)$, retroperitoneal $(18,6 \%)$ y articular $(10,6 \%)$. Los episodios de sangrado a nivel muscular (pared abdominal/torácica) y retroperitoneal fueron mucho más frecuentes en el grupo de HS respecto al grupo de TAO ( 10 y 12 con HS vs. 5 y 2 con TAO; $p<0,001)$. El sangrado en SNC, sin embargo, fue significativamente más frecuente en los pacientes que recibían TAO que aquellos que estaban en tratamiento con HS (20 casos TAO vs de $3 \mathrm{HS}$; $\mathrm{p}<0,001)$.

En el grupo de pacientes con SM y anticoagulación con TAO la media del INR fue de 5,3 (DE $\pm 7,5)$ mientras que en el caso de los pacientes que estaban anticoagulados con HS y que presentaron SM la media del TTPA fue de 2,25 (DE $\pm 0,95)$. Los pacientes tratados con TAO, por tanto, presentaban unos controles de INR por encima de la ratio recomendada (2,5-3,5 veces la ratio normal) en la mayoría de los sangrados mientras que en los pacientes anticoagulados con HS los controles de TTPA estaban dentro del rango, a pesar de presentar sangrado. Existía aparentemente una buena correlación entre SM y exceso de anticoagulación solo en el grupo TA (Tablas I y II).

También las diferencias eran evidentes cuando se analizó el tiempo transcurrido desde el inicio de la anticoagulación hasta el momento del SM. Como es lógico, al ser la anticoagulación con HS un tratamiento inicial durante los primeros días para luego pasar gradualmente al tratamiento de mantenimiento con TAO, la mediana de días entre el inicio de la HS y el SM fue de 7 días (DE $\pm 1,5$ días). En el caso de TAO la mediana de días fue de 570 (DE \pm 120 ) indicando que el episodio de sangrado 
TABLA III

\begin{tabular}{|c|c|c|c|}
\hline Variable & $\begin{array}{c}S N C \\
(n=20)\end{array}$ & $\begin{array}{l}\text { Otros lugares } \\
\quad(n=19)\end{array}$ & P; or (IC95\%) \\
\hline Edad & $68 \pm 11$ & $70 \pm 7$ & 0,46 \\
\hline Sexo masculino & $6(30 \%)$ & $12(63 \%)$ & 0,$05 ; 0,25(0,06-0,9)$ \\
\hline Sangrado previo & $7(35 \%)$ & $8(42 \%)$ & 0,$78 ; 0,7(0,2-2,7)$ \\
\hline \multicolumn{4}{|l|}{ Motivo: } \\
\hline Valvulopatía & $4(20 \%)$ & $4(21 \%)$ & \\
\hline FA & $11(55 \%)$ & $6(32 \%)$ & \\
\hline AVC & $1(5 \%)$ & 0 & \\
\hline TV-TEP & 0 & $6(32 \%)$ & \\
\hline Card.isquemica & $4(20 \%)$ & $3(16 \%)$ & \\
\hline INR o TTPA alto & $13(65 \%)$ & $11(58 \%)$ & 0,$7 ; 0,7(0,2-2,7)$ \\
\hline Exitus atribuible & $7(35 \% 9$ & 0 & 0,008 \\
\hline Tpo.Hasta sangrado & $806 \pm 227$ & $728 \pm 957$ & 0,77 \\
\hline
\end{tabular}

fue muy tardio en este grupo. La Fig 1muestra una curva de Kaplan-Meier del tiempo de sangrado en los dos grupos.

La resolución fue espontánea en 55 pacientes (73\%), siendo necesaria la cirugía en 9 pacientes (12\%). La mayoría de los pacientes intervenidos lo fueron en el grupo de HS (6 pacientes respecto de 3 pacientes en el grupo de TAO; $\mathrm{p}<$ $0,04)$. La mortalidad global (por cualquier causa) fue del $19 \%$ en los casos de SM, si bien la mortalidad directamente atribuible al SM fue del 14,6\% (11 casos) siendo más elevada en el grupo de TAO (8 exitus en el grupo TAO vs. 3 en el grupo $\mathrm{HS}, \mathrm{p}=0,19)$ y estando específicamente concentrada en los casos de sangrado en SNC donde 7/20 (35\%) pacientes tratados con TAO fueron exitus.

Centrando nuestro interés en el grupo de pacientes tratados con TAO y sangrado en SNC por las importantes repercusiones en cuanto a mortalidad. Se realizó un subestudio comparando aquellos pacientes que sangraron en SNC y el resto de pacientes tratados con TAO que sangraron en otras localizaciones. Únicamente, como señala la tabla III, encontramos diferencias en cuanto a mortalidad (7 casos en el grupo de SNC respecto de 0 casos en otras localizaciones; $\mathrm{p}=0,08)$. No existieron diferencias ni en los controles de INR, ni en la variable de sangrado previo, así como tampoco en el resto de variables, excepto en un predominio de mujeres en el grupo de sangrado en SNC.

\section{ESTUDIO COMPARATIVO DE CASOS Y CONTROLES}

Se estudiaron 225 pacientes, 75 casos y 150 controles. De ellos 117 fueron tratados con TAO, siendo 39 de ellos casos y 78 controles. En el estudio comparativo el INR de los casos estaba dentro del rango terapéutico en el episodio del SM en el $38 \%$ de pacientes mientras que los controles tenían el INR dentro del rango en el $75 \%$ de pacientes estudiados $(\mathrm{p}<0,01)$. También existieron diferencias significativas entre casos y controles cuando se analizaba la media de INR entre estos, de forma que los casos presentaron una media de INR de 5,30 y los controles de 2,5 ( $\mathrm{p}<0,001)$.

Se analizaron 108 pacientes anticoagulados con HS, siendo 36 de ellos casos y 72 controles. No se encontraron diferencias significativas entre casos y controles en el momento de SM en sus controles de TTPA, ni tampoco en cuanto a la media del mismo.

Se observó que un $32 \%$ de los casos tenía un antecedente previo de sangrado mientras que tan sólo se observó en un $1,3 \%$ de los controles ( $\mathrm{p}<0,001)$. Además, la historia de sangrado previo fue un factor de riesgo independiente en la aparición de un nuevo episodiode SM posterior en el estudio univariante (RR: 34,5).

Cuando se analizaron estos datos por separado dentro de los grupos de pacientes anticoagulados con TAO por una parte o con HS por otra, se observó que también existían diferencias significativas respecto al sangrado previo.

La mortalidad global fue superior en el grupo de casos respecto de los controles ( $19 \%$ en el grupo de casos respecto de $11 \%$ en el grupo de controles; p : 0,01) (Fig. 3).

\section{DISCUSIÓN}

Nuestros datos revelan que el control de INR por encima de 3,5 estaba directamente relacionado con el riesgo de la aparición de $\mathrm{SM}$ en los pacientes anticoagulados con TAO. La otra variable que en nuestro análisis fue significativa como factor de riesgo independiente de SM fue el hecho de haber presentado un sangrado previo. Esta variable no suele ser analizada en la mayoría de los estudios, pero creemos que puede tener interés clínico ya que define un grupo de riesgo de pacientes sobre los que se puede extremar las precauciones. La mayoría de estos sangrados previos fueron de poca intensidad pero siempre considerados como significativos ( epistaxis,. rectorragia, hematuria, metrorragia etc).

Diversos autores, especialmente en los trabajos más recientes publicados, parecen coincidir con nosotros en el hecho que la intensidad de la descoagulación al menos en el caso de TAO está claramente relacionado con el riesgo de sangrado (14-16). En un estudio realizado en 1997 donde se comparaba la anticoagulación de baja intensidad (ratios entre 1-2,5 de INR) asociada a aspirina a dosis bajas respecto a la descoagulación completa o de alta intensidad (ratio INR entre 2,5-3,5) se observó el doble de episodios de sangrado en el grupo de acenocumarol y anticoagulación de alta intensidad (16).

Así, parece haber una relación evidente entre los episodios de sangrado y el control del INR, (en los pacientes tratados con TAO) de forma que Landefeld y cols. (17) encontraron que por cada 1 de incremento en la cifra del INR la Odd ratio relacionada con el riesgo de sangrado aumentaba un $8 \%$. Van der Meer y cols. (8) también coincidieron con este hecho en su serie de pacientes así como el estudio ISCOAT (18) o el trabajo de Finh y cols. (19) que coinciden en valorar que con ratios de INR superiores a 4,5 el riesgo de sangrado fue significativamente mayor. En el estudio de Casais y cols. (10) finalmente se encontró un riesgo significativo de sangrado mayor cuando la ratio de INR era superior a 6.

Recientemente una publicación de Koo S y cols. (20) presenta un estudio prospectivo de 101 paciente con sangrado mayor que viene también a cofirmar estos datos dado que la excesiva anticoagulación fue un factor de riesgo independiente predictora de mortalidad. En este estudio y respecto de los sangrados intracraneales resulta interesante comentar que si bien esta complicación aumenta en el caso de excesiva descoagulación, un elevado porcentaje de sus pacientes con sangradoen SNC tenían controles correctos. 
Otros autores han afirmado que la polimedicación frecuente en estos pacientes puede también facilitar el sangrado especialmente cuando se asocian con antiagregantes como la aspirina o los antiinflamatorios esteroideos o no esteroideos (AINES), etc. $(21,22)$. Los estudios de Casais y cols. $(9,10)$ y el nuestro no encontraron una relación significativa entre estos tratamientos cuando se analizaron los episodios de SM. Parece, no obstante, interesante seguir intruyendo a los pacientes para que frente a cualquier nuevo tratamiento que se les instauren consulten con su hematologo con el objetivo de evitar interacciones con los anticoagulantes orales.

\section{Bibliografía}

1. Landefeld CS, Beyth RJ. Anticoagulant-related bleeding clinical epidemiology, prediction and prevention. Am J Med 1993; 95: 315-28.

2. Levine MN, Raskob G, Hirsh J. Hemorrhage complications of longterm anticoagulant therapy. Chest 1995; 108: 291s-301s.

3. Fihn SD, Mc Donell M, Martin D, Henikoff J, Vermes D, Kent D, White RH. Risk factors for complications of chronic anticoagulation: A multicenter study. Ann Intern Med 1993; 118: 511-20.

4. Debray M, Pautas E, Couturier P, Franco A, Siguret V. Oral anticoagulants in the elderly. Rev Med Interne 2003; 24: 107-17.

5. Poli D, Antonucci E, Lombardi A, Boddi V, Gensini GF, Abbate R, et al. Low rate of bl.eeding and thrombotic complications of oral anticoagulant therapy independent of age in the real-practice of an anticoagulation clinic. Blood Coagul Fibrinolysis 2003;14: 269-75.

6. Wells PS, Forgie MA, Simms M, Greene A, Touchie D, Lewis G, Anderson J, Rodger MA. The outpatient bleeding risk index: Validation of a tool for predicting bleeding rates in patients treated for deep venous thrombosis and pulmonary embolism.

7. Freixa R, Blanch P, Ibernon M, Padro J, Delso J, Sobrepera JL, et al. Identification of factors responsible for oral over-anticoagulation in outpatients with heart disease. Rev Esp Cardiol 2003; 56: 65-72.

8. Van der Meer FJM, Rosendaal FR, Vandenbroucke JP, Brie"t E. Assessment of a bleeding risk index in two cohorts of patients treated with oral anticoagulants. Thromb Haemost 1996; 76: 12-16.

9. Casais P, Sánchez Luceros A, Meschengieser S, Fondevila C. Santarelli M, Lazzari M. Bleeding risk factors in chronic oral anticoagulation with acenocoumarol Am J hematol 2000; 63: 192-6.

10. Casais P, Luceros AS, Meschengieser S, Bermejo E, Lazzari MA.INR variability in anticoagulation with acenocoumarol: Is it useful for identifying patients at risk of bleeding and thrombosis? Haematologica 1999; 84: 753-4.

11. Simonneau G, Sors H, Charbonnier B, Page Y, Laaban JP, Azarian R, et al. A comparison of low-molecular-weight heparin with unfractionated heparin for acute pulmonary embolism. The THESEE Study Group. Tinzaparine ou Heparine Standard: Evaluations dans l'Embolie Pulmonaire. N Engl J Med 1997; 337: 663-9.
Como conclusiones final en nuestra experiencia, la anticoagulación con TAO presenta su principal complicación (sangrado) en pacientes con anticoagulación excesiva detectada por la práctica periódica del INR. Una monitorización más frecuente y/o la vigilancia más estricta en aquellos pacientes en los que el INR es más dificil de controlar podría ser la estrategia a seguir. El estudio de los factores de riesgo para detectar los pacientes que presentan mayor dificultad para mantener el INR dentro de los controles podría ser también una linea de estudio interesante si bien difícil de realizar a nuestro juicio. Finalmente los pacientes con sangrado previo deben ser estrictamente seguidos a la luz de nuestros datos.

12. Wester JP, de Valk HW, Nieuwenhuis HK, Brouwer CB, van der Graaf Y, Meuwissen OJ, et al. Risk factors for bleeding during treatmen of acute venous thromboembolism. Thromb Haemost 1996; 76: 682-8

13. Gitter MJ, Jaeger TM, Petterson TM, Gersh BJ, Silverstein MD. Bleeding and thromboembolism during anticoagulant therapy: A populationbased study in Rochester, Minnesota. Mayo Clin Proc 1995; 70: 725 733.

14. Hylek EM, Singer DE. Risk factors for intracranial hemorrhage in outpatients taking warfarin. Ann Intern Med 1994; 120: 897-902.

15. Toulemonde $\mathrm{F}$. The role of individual risk factors in anticoagulantassociated hemorrhages. Sem Thromb Hemostas 1996; 22: 53-60.

16. Meschengieser SS, Fondevila CG, Frontroth J, Santarelli MT, Lazzari MA. Low intensity oral anticoagulation plus low-dose aspirin versus high-intensity oral anticoagulation alone: A randomized trial in patients with mechanical prosthetic heart valves. J Thorac Cardiovasc Surg 1997; 113: 910-6.

17. Landefeld CS, Beyth RJ. Anticoagulant-related bleeding: Clinical epidemiology, prediction, and prevention. Am J Med 1993; 95: 315-28.

18. Palareti G, Leali N, Coccheri S, Poggi M, Manotti C, D’Angelo A, et al On behalf of the Italian Study on Complications of Oral Anticoagulant Therapy. Bleeding complications of oral anticoagulant treatment: An inception-cohort, prospective collaborative study (ISCOAT). Lancet 1996; 348: 423-8.

19. Fihn SD. Aiming for safe anticoagulation. N Engl J Med 1995; 333: 54-5.

20. Koo S, Kucker N, Nguyen PL, Fanikos J, Marks PW, Goldhaber SZ The effect of excessive anticoagulation on mortality and morbility in hospitalized patients with anticoagulant-related major hemorrhage. Arch Intern Med 2004; 164: 1557-60.

21. Samoczi M, Farkas A, Sipos E, Tarjan J. Adverse effects of combined use of acenocoumarol and acetylsalicylic acid after myocardial infarct and unstable angina. Orv Hetil 1995; 136: 177-9.

22. Visser LE, Penning-van Beest FJ, Kasbergen AA, De Smet PA, Vulto AG, Hofman A, et al. Overanticoagulation associated with combined use of antifungal agents and coumarin anticoagulants. Clin Pharmacol Ther 2002; 71: 496-502. 